\title{
Silencing of Long non-coding RNA Plasmacytoma variant translocation 1 inhibits the proliferation, migration, invasion and fibrosis of high glucose- induced mouse mesangial cells via targeting microRNA-93-5p
}

Jianzhou Li

Caoxian People's Hospital

Qing Zhao

Caoxian People's Hospital

Xiaohong Jin

Caoxian People's Hospital

Yanhua Li

First People's Hospital of Jinan City

Jian Song ( $\nabla$ songjian137ql@163.com )

Qilu Hospital of Shandong University https://orcid.org/0000-0002-1361-4410

Research article

Keywords: diabetic nephropathy; LncRNA-PVT1; miR-93-5p; PI3K/Akt/mTOR pathway; mouse mesangial cells

Posted Date: December 3rd, 2019

DOI: https://doi.org/10.21203/rs.2.14072/v2

License: (c) (i) This work is licensed under a Creative Commons Attribution 4.0 International License.

Read Full License 


\section{Abstract}

Background: This study aimed to investigate the regulatory role of Long non-coding RNA plasmacytoma variant translocation 1 (PVT1) on the biological processes of high glucose (HG)-induced mouse mesangial cells (MMCs). Methods: PVT1 expression in diabetic nephropathy (DN) mice and HG-induced MMCs was detected by qRT-PCR. PVT1 was silenced in HG-induced MMCs by the transfection of PVT1 siRNA (si-PVT1). EdU and Colony formation, Annexin V-PI staining, Muse cell cycle, Scratch, and Transwell assay were performed to detect the cell proliferation, apoptosis, cell cycle, migration, and invasion, respectively. The contents of fibrosis factors in cell-culture supernatants were detected by ELISA. Western blot was performed to detect the expression of factors involved in apoptosis, cell cycle, migration and invasion, fibrosis, and PI3K/Akt/mTOR pathway. In addition, the targeting relation between miR-93-5p and PVT1 was predicted by StarBase3.0 and identified by Dual-luciferase reporter gene assay. Results: PVT1 was overexpressed in DN kidney tissues and HG-induced MMCs. HG-induced MMCs exhibited significantly increased EdU positive cells, cell colonies, $S$ and G2/M phase cells, migration and invasion ability, and contents of fibrosis factors, as well as significantly decreased apoptosis rate compared with NG-induced MMCs. HG significantly up-regulated Bcl-2, CyclinD1, CDK4, N-cadherin, vimentin, Col. IV, FN, TGF- $\beta 1$ and PAI-1, and down-regulated Bax, cleaved caspase-3, cleaved PARP, and Ecadherin in MMCs. Silencing of PVT1 eliminated the effects of HG in MMCs and blocked PI3K/Akt/mTOR pathway. MiR-93-5p was a target of PVT1, which eliminated the effects of PVT1 on HG-induced MMCs. Conclusions: PVT1 silencing inhibited the proliferation, migration, invasion and fibrosis, promoted the apoptosis, and blocked PI3K/Akt/mTOR pathway in HG-induced MMCs via up-regulating miR-93-5p.

\section{Introduction}

Background: Diabetic nephropathy (DN), characterized by decreased glomerular filtration, proteinuria, and renal fibrosis is a common diabetes-associated disease [1]. DN affects more than $40 \%$ diabetic patients worldwide, and leads to millions of deaths due to end-stage kidney disease [2]. The occurrence of DN attributes to diverse factors, such as hyperglycemia, accumulation of advanced glycosylation products, and activation of cytokines [3]. Although many attempts have been made to the clinical treatment of DN $[4,5]$, the modern medical treatment is still unable to completely prevent the development and deterioration of DN due to the insufficient understanding of the pathological mechanisms involving DN [6].

Long non-coding RNAs (IncRNAs) are important transcripts that take part in the regulation of disease pathways [7-10]. The dys-regulation of IncRNAs has been widely reported in various diseases, including DN $[11,12]$. Plasmacytoma variant translocation 1 (PVT1) is an important IncRNA regulator in diabetes [13]. It has proved that autophagy ameliorates cognitive impairment in diabetic mice through activating PVT1 [14]. Type 1 diabetes-induced end-stage renal disease is closely associated with the variants in PVT1 [15]. PVT1 mediates the accumulation of extracellular matrix in DN [16]. LncRNAs can modulate the translation and degradation of mRNAs through interacting with microRNAs (miRs) [17]. The biological function of PTV1 during the disease process is realized by targeting specific miRs [18]. Six locations on 
PVT1 are revealed on miR-1204, -1205, -1206, 1207-3p, -1207-5p, and -1208 [19]. The mediation effect of PTV1 during extracellular matrix accumulation in kidney cells of DN is realized by targeting miR-1207-5p [20]. MiR-93 is an onco-miR in cancer [21], that is also a regulator in DN [22]. It has proved that the serum miR-93 is low-expressed in DN patients [23]. Comparative miR expression profile arrays showed that miR93 is a signature miR in hyperglycemic condition [24]. Up-regulation of miR-93 inhibits epithelial mesenchymal transformation and renal fibrogenesis in TGF- $\beta 1$-induced HK2 cells [25]. Although the biological functions of PTV1 and miR-93 in DN have been mentioned by previous studies, the detailed molecular mechanism of PTV1 involving miR-93 in DN remains unclear.

Objectives: In this study, the regulatory role of PVT1 on the proliferation, apoptosis, cell cycle, migration, invasion, and fibrosis of high glucose (HG)-induced mouse mesangial cells (MMCs) was evaluated. The regulatory mechanism of PVT1 involving miR-93 was analyzed. This study may lay a novel theoretical basis for the treatment of DN.

\section{Methods}

\section{Animal}

A total of 10 SPF DN mice (4-week-old, Strain: BKS.Cg-Leprdb/Leprdb/J, db/db, 20-30 g) and 10 normal mice (4-week-old, db/m, 20-30 g) were obtained from the Model Animal Research Center of Nanjing University (Nanjing, China). Mice were fed in a standalone environment at $22^{\circ} \mathrm{C}$ and $50 \%$ relative humidity under the alternating day and night of $12 \mathrm{~h} / 12 \mathrm{~h}$. After 4 days of feeding with standard diet (acclimatization), mice were anesthetized by intraperitoneal injection of $50 \mathrm{mg} / \mathrm{kg}$ sodium pentobarbital, and then sacrificed by cervical dislocation. The kidney tissues were stripped and frozen in liquid nitrogen. This study was approved by the local ethics committee of our hospital. All animal experiments were performed in accordance with the care and use of laboratory animals (National Institutes of Health, USA).

\section{Cell culture}

MMCs (SV40 MES13, established from the kidney of a mouse transgenic for the SV40 early region) were purchased from the Cell Bank of the Chinese Academy of Sciences (Shanghai, China). MMCs were cultured in a mixture of DMEM containing 10\% FBS and Ham's F12 medium (3: 1) containing $14 \mathrm{mM}$ HEPES, and maintained in a humidified incubator (MCO-15AC, SANYO) with $5 \% \mathrm{CO}_{2}$ at $37^{\circ} \mathrm{C}$. The medium was refreshed every $48 \mathrm{~h}$, and cells were passaged at $80 \%$ confluence. Logarithmic growth phase cells at the third passage were used for transfection.

\section{Cell treatment and transfection}

PVT1 siRNA (si-PVT1) and siRNA negative control (si-NC) were synthesized by Shenggong Bioengineering Co., Ltd. (Shanghai, China) (Table 1). MiR-93-5p mimics, miR-93-5p inhibitor, mimics negative control (mimics-NC) and inhibitor negative control (INC) were purchased from Jima Pharmaceutical Technology Co., Ltd. (Shanghai, China). Cells were transfected with the above agents by 
using a Lipofectamine kit (Invitrogen). Cells without transfection were considered as the control. The transfection efficiency was identified by qRT-PCR. After the transfection for $48 \mathrm{~h}$, cells were incubated in DMEM containing $5.55 \mathrm{mmol} / \mathrm{L}$ (normal physiological environment, NG group) or $30 \mathrm{mmol} / \mathrm{L}$ D-Glucose (diabetic physiological environment, HG group) for another $48 \mathrm{~h}$. Cells were collected for the following assay.

qRT-PCR

Total RNA was extracted from MMCs by using TRIZOL reagent (Invitrogen), and then reverse-transcribed on a SimpliAmp PCR instrument (Applied Biosystems, Foster City, CA, USA). The qRT-PCR was performed on an ABI7500 Sequence Detection System (Applied Biosystems). The PCR program included 40 cycles of $95^{\circ} \mathrm{C}$ for $10 \mathrm{~min}, 95^{\circ} \mathrm{C}$ for $10 \mathrm{~s}, 60^{\circ} \mathrm{C}$ for $20 \mathrm{~s}$ and $72^{\circ} \mathrm{C}$ for $34 \mathrm{~s}$. The relative mRNA expression level was analyzed by the $2^{-\Delta \Delta C t}$ method [26]. Oligonucleotide primers were synthesized by Biotechnology Bioengineering Co., Ltd. (Shanghai, China), and the primer sequences were shown in Table 1.

\section{Western blot}

Total proteins were extracted from cells using lysis buffer, and quantified using a BCA kit (Invitrogen). Then, $20 \mu \mathrm{g}$ protein samples were run in a 10\% SDS-PAGE, and transferred onto PVDF membrane. Afterwards, the membrane was blocked with $5 \%$ skim milk in TBST solution for $2 \mathrm{~h}$, and incubated with primary antibody overnight at $4^{\circ} \mathrm{C}$. The antibodies included E-cadherin $(1: 1000,3195, \mathrm{CST}), \mathrm{N}$-cadherin (1:1000,13116, CST), Vimentin (1:1000, 5741, CST), GAPDH (1: 1000, 5174, CST), Col. $\otimes(1: 10000$, ab6586, Abcam), FN (1:10000, ab2413, Abcam), TGF- 31 (1:10000, ab92486, Abcam), PAl-1 (1:10000, ab66705, Abcam), Bcl-2 (1:1000, ab196495, Abcam), Bax (1:1000, ab199677, Abcam), cleaved caspase-3 (1:1000, ab49822, Abcam), cleaved PARP (1:1000, ab32064, Abcam), CyclinD1 (1:5000, ab226977, Abcam), CDK4 (1:3000, ab137675, Abcam), PI3K (1:1000, 4292, CST), p-PI3K (1:1000, 4228, CST), Akt (1:1000, 9272, CST), p-Akt (1:1000, 4060, CST), mTOR (1:1000ه2972هCST), and p-mTOR(1:1000, 2971, CST). GAPDH was used as the internal control. After 3 times of washing with TBST, the membrane was incubated with HRP-labelled secondary antibody $\left(1: 1000\right.$, Sigma) for $2 \mathrm{~h}$ at $25^{\circ} \mathrm{C}$. An enhanced chemiluminescence Plus (Thermo Fisher) was used to visualize the protein bands. The gray value was measured by using a Lab Works 4.5 software. Western blot was performed in three independent repetitions, and the representative images were shown.

\section{EdU and Colony formation assay}

An EdU cell proliferation detection kit (Ruibo Biotechnology Co., Ltd., Guangzhou, China) was used for EdU assay (cell proliferation). Briefly, cells were labeled with EdU, fixed with $4 \%$ paraformaldehyde, and then stained with DAPI. The EdU fluorescence (red) was detected by a laser confocal microscope (NIKON A1, Japan).

For Colony formation assay, cells were seeded into 6-well plates (300 cells/well) and cultured for 2 weeks. Cells were then fixed with $4 \%$ paraformaldehyde for $10 \mathrm{~min}$ and stained with crystal violet for $15 \mathrm{~min}$. The 
stained colonies were observed under an inverted microscope (Olympus Ckx53, Japan), and counted using an ImageJ (1.48 V) software.

\section{Annexin V-PI assay}

An Annexin V-PI kit (Invitrogen) was used for the detection of cell apoptosis. Briefly, cells were stained with Annexin V-EGFP and PI for 15 min under darkness at $25^{\circ} \mathrm{C}$. The apoptosis rate was then analyzed on a MUSETM cytometer (EMD Millipore, USA).

\section{Cell cycle analysis}

A MuseTM Cell Cycle Reagent (Invitrogen) was used for the detection of cell cycle. Briefly, cells were fixed in $70 \%$ ethanol, and incubated with MuseTM Reagent in the dark for 30 min at $25^{\circ} \mathrm{C}$. The cell cycle was then analyzed on a MUSETM cytometer (EMD Millipore).

\section{Scratch assay}

Cells were seeded into 6-well plate, and cultured in serum-free DMEM until 90\% confluence. A scratch wound was then made in each well with a pipet tip. Cell debris was removed by washing with PBS. After $48 \mathrm{~h}$ of culturing in serum-free DMEM, the scratch width was photographed under an inverted microscope (Olympus $\mathrm{Ckx} 53)$. The migration rate $=(1-$ scratch width at measurement $/$ initial scratch width $) \times 100 \%$.

\section{Transwell assay}

The cell invasion was detected by using transwell chambers (Corning Corporation, Midland, MI, USA). Briefly, cells were seeded into Matrigel-coated upper chamber. The lower chamber was filled with DMEM. After $48 \mathrm{~h}$ of incubation, cells on the lower chamber were fixed with $4 \%$ paraformaldehyde for $15 \mathrm{~min}$ and stained with $0.1 \%$ crystal violet for 20 min. The stained cells were observed under an inverted microscope (Olympus Ckx53), and counted randomly at five fields of views at $200 \times$ magnifications.

\section{ELISA for fibrosis factors}

Cells were cultured for $48 \mathrm{~h}$ after transfection, and the cell-culture supernatants were collected. The contents of Col. IV, PAI-1, FN and TGF- $\beta 1$ in the supernatants were detected by using ELISA kits (BioSource International, Camarillo, CA, USA) in accordance with the manufacturers' instructions.

\section{Dual-Luciferase reporter gene (DLR) assay}

The binging site of PVT1 in miR-93-5p was predicted by StarBase3.0 software. A DLR kit (Promega, USA) was used to identify the relationship between PVT1 and miR-93-5p. The mutant sequence (MT) and the wild sequence (WT) of PVT1 were amplificated according to the binging sequences, and then cloned into the pmirGLO luciferase vector. HEK-293T cells were then co-transfected with WT/MT and miR-93-5p

mimics/miR-93-5p mimics NC (Shanghai Jima Pharmaceutical Technology Co., Ltd.), and grouped as MT 
+ mimics, MT + NC, WT + mimics, and WT + NC group. After $48 \mathrm{~h}$ of transfection, the fluorescence was measured on a Microplate Reader (Invitrogen).

\section{Statistical analysis}

All assays were performed in triplicate. Data were expressed as mean \pm standard deviation (SD). All statistical analyses were performed by SPSS 20.0 and GraphPad.Prism 5.01 statistical software. The data among multi-groups were analyzed by One-Way ANOVA, followed by Tukey's test (between two groups). $\mathrm{P}<0.05$ represented statistically significant.

\section{Results}

\section{PVT1 was up-regulated in DN mice and HG-induced MMCs}

The expression of PVT1 was detected in kidney tissues of DN mice and HG-induced MMCs by qRT-PCR. PVT1 expression was significantly higher in kidney tissues of $\mathrm{db} / \mathrm{db}$ mice (DN) than that in tissues of $\mathrm{db} / \mathrm{m}$ mice (Normal) $(\mathrm{P}<0.05)$ (Figure $1 \mathrm{~A})$. In addition, PVT1 was significantly up-regulated in HGinduced MMCs compared with NG-induced MMCs $(P<0.05)$ (Figure 1B).

\section{Silencing of PVT1 inhibited the proliferation of HG-induced MMCs}

PVT1 was silenced in HG-induced MMCs by the transfection of siPVT1. The mRNA expression of PVT1 in the $H G+$ siPVT1 group was significantly lower than that in the HG group $(P<0.05)$. The expression of PVT1 was not significantly different between the HG group and the HG + siNC group (Figure 2A). EdU assay showed that the percentage of EdU cells in the HG group was significantly higher than that in the NG group $(P<0.05)$. The percentage of EdU cells was significantly decreased in the HG + siPVT1 group compared with that in the $H G$ group $(P<0.05)$ (Figure $2 B$ ). Colony formation assay showed consistent results with EdU assay $(P<0.05)$ (Figure $2 C)$. In addition, the cell apoptosis was opposite to the cell proliferation detected above $(P<0.05)$ (Figure 2D). The expression of Bax, cleaved caspase-3, and cleaved PARP in the HG group was significantly lower than that in the NG group. The expression of Bax, caspase-3, and PARP was significantly increased in the HG + siPVT1 group compared with that in the HG group $(\mathrm{P}<0.05)$. The expression change of $\mathrm{Bcl}-2$ was opposite to that of $\mathrm{Bax}(\mathrm{P}<0.05)$ (Figure $2 \mathrm{E})$.

\section{Silencing of PVT1 influenced the cell cycle of HG-induced MMCs}

The percentages of cells in S and G2/M phase were significantly higher and the percentage of cells in G0/G1 phase was significantly lower in the HG group than that in the NG group (all $P<0.05$ ). The transfection of siPVT1 eliminated the effects of HG on the cell cycle of MMCs (all $P<0.05$ ), suggesting that PVT1 silencing might block the cell cycle of HG-induced MMCs in G0/G1 phase (Figure 3A and B). The expression of Cyclin D1 and CDK4 in HG-induced MMCs was further detected by qRT-PCR and Western blot. CyclinD1 and CDK4 in the HG group were significantly up-regulated compared with the NG group at both the mRNA and protein level (all $\mathrm{P}<0.05$ ). The mRNA and protein expression of CyclinD1 
and CDK4 were decreased in the HG + siPVT1 group compared with those in the HG group (all P > 0.05) (Figure 3C-E).

\section{Silencing of PVT1 inhibited the migration and invasion of HG-induced MMCs}

Scratch and Transwell assay showed that the cell migration and invasion ability were significantly enhanced in the HG group compared with the NG group (all $P<0.05$ ). The transfection of siPVT1 inhibited the migration and invasion ability of HG-induced MMCs (all $P<0.05$ ) (Figure 4A-B). Western blot showed that the expression of E-cadherin was significantly lower, and the expression of $\mathrm{N}$-cadherin and vimentin was significantly higher in the HG group than that in the NG group (all $P<0.05$ ). The transfection of siPVT1 significantly reversed the expression of E-cadherin, $\mathrm{N}$-cadherin and vimentin in HGinduced MMCs (all P < 0.05) (Figure 4C).

\section{Silencing of PVT1 inhibited the fibrosis of HG-induced MMCs}

The contents of fibrosis factors in the supernatant of MMCs were detected by ELISA. The contents of Col. IV, FN, TGF- $\beta 1$ and PAI-1 in the HG group were significantly higher than those in the NG group (all $\mathrm{P}<$ 0.05). The contents of fibrosis factors in the HG+siPVT1 group were significantly decreased compared with those in the HG group (all $P<0.05$ ) (Figure 5 A-D). In addition, Western blot showed that the expression of fibrosis factors was consistent with the ELISA results (all $P<0.05$ ) (Figure $5 \mathrm{E}$ ).

\section{MiR-93-5p was the target gene of PVT1}

A binging site of PVT1 at miR-93-5p was predicted by StarBase3.0 software (Figure 6A). Because miR-93 plays an important role in the progression of DN, the targeting relation between miR-93-5p and PVT1 was further analyzed. DLR assay showed that the luciferase activity in the WT + mimics group was significantly lower than that in the WT + NC group $(\mathrm{P}<0.05)$ (Figure $6 \mathrm{~B})$. The mRNA expression of PVT1 was significantly higher in the inhibitor group and significantly lower in the mimics group than that in the Mock group (all P < 0.05). The expression of PVT1 was not significantly influenced by the transfection of mimics-NC and INC. In addition, the expression of miR-93-5p in the HG group was significantly lower than that in the NG group $(P<0.05)$. The transfection of siPVT1 significantly up-regulated miR-93-5p in HGinduced MMCs $(P<0.05)$ (Figure 6C and D).

\section{MiR-93-5p eliminated the role of PVT1 in HG-induced MMCs}

MiR-93-5p inhibitor was used to silence miR-93-5p in HG-induced MMCs. The mRNA expression of miR93-5p in the inhibitor group was significantly down-regulated compared with that in the Mock group $(\mathrm{P}<$ 0.05). The expression of miR-93-5p was not significantly different between the INC group and the Mock group (Figure 7A). The miR-93-5p inhibitor and siPVT1 were then con-transfected into HG-induced MMCs. The cell proliferation, migration, invasion, and fibrosis ability were promoted by the transfection of miR93-5p inhibitor, and inhibited by the transfection of siPVT1 (all P < 0.05). The transfection of miR-93-5p inhibitor significantly eliminated the effects of siPVT1 on the above biological processes of HG-induced MMCs (all $P<0.05)$ (Figure 7B-E). 
The expression of PI3K/Akt/mTOR pathway related proteins was detected by Western blot. The expression of p-PI3K/PI3K, p-Akt/Akt, and p-mTOR/mTOR was higher in the siNC + miR-93-5p inhibitor group and was lower in the siPVT1 + INC group than that in the siNC + INC group (all $P<0.05$ ). The blocking effect of siPVT1 on PI3K/Akt/mTOR pathway was eliminated by miR-93-5p inhibitor (all $\mathrm{P}<$ 0.05) (Figure 8).

\section{Discussion}

DN is one of the most common conditions found in patients with renal disease. Although PVT1 has been proved to be associated with DN, the detailed molecular mechanism of PVT1 in DN progression remains unclear. Here, PVT1 was found to be overexpressed in kidney tissues of ND mice and HG-induced MMCs. Silencing of PVT1 inhibited the proliferation, migration, invasion and fibrosis, promoted the apoptosis, and blocked PI3K/Akt/mTOR pathway in HG-induced MMCs. In addition, PVT1 negatively regulated its target miR-93-5p in MMCs. MiR-93-5p eliminated the effects of PVT1 on the biological processes of HGinduced MMCs.

Mesangial cells (MCs) are key functional cells in the development of DN, which can mediate the glomerular filtration rate [27]. Over-proliferation of MCs is involved in the development of NC [28]. IncRNAs play important regulatory roles in the progression of DN. Gao et al. proved that IncRNAASncmtRNA-2 is up-regulated in DN kidney tissues and HG-treated MCs, and its overexpression promotes the glomerular fibrosis in DN [29]. Li et al. showed that IncRNA-1700020114Rik contributes to the fibrosis of MMCs cultured with HG medium [30]. Wang et al. proved that overexpression of IncRNAENSMUST00000147869 reversed DN-induced proliferation and fibrosis of MCs [31]. The cell proliferation and fibrosis can also be regulated by IncRNA-CYP4B1-PS1-001 in DN [32]. Actually, as a member of IncRNAs, PVT1 also participates in the cell proliferation, migration, invasion and fibrosis in diseases [33, 34]. Huang et al. showed that PVT1 overexpression promotes the cell migration and invasion, and is a potential prognostic biomarker of small-cell lung cancer [35]. Zhao et al. proved that PVT1 promotes the migration and proliferation of pancreatic cancer cells via targeting miR-448 [36]. Alvarez et al. proved that PVT1 is up-regulated for 5 -folds in response to hyperglycemia, and PVT1 can mediate the accumulation of extracellular matrix in DN [37]. Here, PVT1 was up-regulated in DN kidney tissues and HG-induced MMCs. Silencing of PVT1 significantly inhibited the proliferation of HG-induced MMCs through blocking the cell cycle at G0/G1 phase, as well as the migration, invasion and fibrosis of HG-induced MMCs. We speculate that PVT1 silencing may contribute to the remission of DN through mediating the above cellular biological processes.

Some miRNAs mapping to the PVT1 locus play important roles in disease progression [38]. LncRNAMEG3 inhibits the proliferation of glioma cells by targeting miR-93 and blocking PI3K/AKT pathway [39]. MiR-93 is a metabolic/epigenetic switch in diabetes, which connects the metabolic state and chromatin remodeling [40]. Zou et al. indicated that the plasma level of miR-93 was a potential predictor for high risk 
of diabetes-related diseases [41]. Furthermore, the regulatory pathway of PI3k/Akt/mTOR is important in the biological process of DN [42]. The phosphorylation of AKT and mTOR is a landmark reaction of DN [43]. Akt/mTOR pathway is a potential target to prevent renal fibrosis in DN [44]. MiR-196b and miR-451 influence the cell proliferation and invasion through regulating PI3K/AKT/mTOR pathway in cancer cells $[45,46]$. In this study, miR-93-5p inhibitor counteracted the inhibitory effect of siPVT1 on PI3K/Akt/mTOR pathway. We speculate that the PVT1 may activate the PI3K/Akt/mTOR pathway through targeting miR93-5p, thereby regulating the proliferation, apoptosis, migration, invasion and fibrosis of HG-induced MMCs. However, our findings are limited in the biological processes of HG-induced MMCs. Further researches on the regulatory effect and mechanism of PVT1 in animal models of DN are still needed.

\section{Conclusions}

Silencing of PVT1 inhibited the proliferation, migration, invasion and fibrosis, promoted the apoptosis, and blocked PI3K/Akt/mTOR pathway in HG-induced MMCs through targeting miR-93-5p. PVT1 was speculated as a potential therapeutic target for DN.

\section{List Of Abbreviations}

LncRNA- plasmacytoma variant translocation 1 (PVT1)

Mouse mesangial cells (MMCs)

diabetic nephropathy (DN)

real-time fluorescence quantitative polymerase chain reaction (RT-qPCR)

enzyme-linked immunosorbent assay (ELISA)

end-stage renal disease (ESRD)

microRNAs (miRNAs)

PVT1 siRNA (si-PVT1)

negative control group (si-NC)

blank control group (Mock)

si-PVT1 experimental group (siPVT1)

control group (NG)

high glucose group ( $\mathrm{HG})$

high glucose negative control group ( $\mathrm{HG}+\mathrm{siNC})$ 
high glucose siPVT1 group (HG + siPVT1)

\section{Declarations}

\section{Ethics approval and consent to participate}

This study was approved by the ethics committee of Chongqing Medical University, and all experiments were performed in accordance with the guide for the care and use of laboratory animals established by the United States National Institutes of Health.

\section{Consent to publish}

All contributing authors have agreed to submit this manuscript and all authors approved to publish this study.

\section{Availability of data and materials}

The datasets used and/or analyzed during the current study are available from the corresponding author on reasonable request.

\section{Competing interests}

The authors declare that they have no competing interests.

Funding: None.

\section{Authors'Contributions}

JZL, QZ and XHJ were involved in the conception and design of the study, and development of the manuscript. YHL was participated in the conduct of the experiment and collection of data. JS participated in the drafting or critical revision of the manuscript. All authors read and approved the final manuscript.

Acknowledgments: None.

\section{References}

1. Ritz E: Diabetic nephropathy. Saudi J Kidney Dis Transp/ 2006, 17(4):481-490.

2. Yan D, Choi ME: Autophagy in diabetic nephropathy. J Endocrinol 2015, 224(1):R15.

3. Su Y: Recent advances in understanding the biochemical and molecular mechanism of diabetic nephropathy. Biochem Biophys Res Commun 2013, 433(4):359-361.

4. Huang Z, Mou Y, Xu X, Zhao D, Lai Y, Xu Y, Chen C, Li P, Peng S, Tian J: A Novel Derivative of Bardoxolone Methyl Improves Safety for Treatment of Diabetic Nephropathy. J Med Chem 2017, 60(21):acs.jmedchem.7b00971. 
5. Egido J, Rojasrivera J, Mas S, Ruizortega M, Sanz AB, Gonzalez PE, Gomezguerrero C: Atrasentan for the treatment of diabetic nephropathy. Expert Opin Investig Drugs 2017, 26(6).

6. Correa-Rotter R, Gonzã I-ML: Early detection and prevention of diabetic nephropathy: a challenge calling for mandatory action for Mexico and the developing world. Kidney Int 2005, 68(98):S69.

7. UA Ø, Derrien T, Beringer M, Gumireddy K, Gardini A, Bussotti G, Lai F, Zytnicki M, Notredame C, Huang Q: Long noncoding RNAs with enhancer-like function in human cells. Med Sci (Paris) 2010, 143(1):46-58.

8. Maass PG, Rump A, Schulz H, Stricker S, Schulze L, Platzer K, Aydin A, Tinschert S, Goldring MB, Luft FC: A misplaced IncRNA causes brachydactyly in humans. J Clin Invest 2012, 122(11):3990-4002.

9. Kotake Y, Nakagawa T, Kitagawa K, Suzuki S, Liu N, Kitagawa M, Xiong Y: Long non-coding RNA ANRIL is required for the PRC2 recruitment to and silencing of p15(INK4B) tumor suppressor gene. Oncogene 2011, 30(16):1956.

10. Khaitan D, Dinger ME, Mazar J, Crawford J, Smith MA, Mattick JS, Perera RJ: The melanomaupregulated long noncoding RNA SPRY4-IT1 modulates apoptosis and invasion. Cancer Research 2011, 71(11):3852.

11. Hu M, Wang R, Li X, Fan M, Lin J, Zhen J, Chen L, Lv Z: LncRNA MALAT1 is dysregulated in diabetic nephropathy and involved in high glucose-induced podocyte injury via its interplay with $\beta$-catenin. $J$ Cell Mol Med 2017, 21(11):2732-2747.

12. The efficiency of serum IncRNA GAS5/miR-21 as biomarkers in patients with diabetes and diabetic nephropathy. China Medical Abstracts(Internal Medicine) 2018, v.35(1):55-56.

13. Hanson RL, Craig DW, Millis MP, Yeatts KA, Kobes S, Pearson JV, Lee AM, Knowler WC, Nelson RG, Wolford JK: Identification of PVT1 as a candidate gene for end-stage renal disease in type 2 diabetes using a pooling-based genome-wide single nucleotide polymorphism association study. Diabetes 2007, 56(4):975.

14. Li Z, Hao S, Yin H, Gao J, Yang Z: Autophagy ameliorates cognitive impairment through activation of PVT1 and apoptosis in diabetes mice. Behav Brain Res 2016, 305:265-277.

15. Millis MP, Bowen D, Kingsley C, Watanabe RM, Wolford JK: Variants in the Plasmacytoma Variant Translocation Gene (PVT1) Are Associated With End-Stage Renal Disease Attributed to Type 1 Diabetes. Diabetes, 56(12):3027-3032.

16. M Lucrecia A, Distefano JK: Functional characterization of the plasmacytoma variant translocation 1 gene (PVT1) in diabetic nephropathy. Plos One 2011, 6(4):e18671.

17. Wu T, Qu L, He G, Tian L, Li L, Zhou H, Jin Q, Ren J, Wang Y, Wang J: Regulation of laryngeal squamous cell cancer progression by the IncRNA H19/miR-148a-3p/DNMT1 axis. Oncotarget, 7(10).

18. He RQ, Qin MJ, Lin P, Luo YH, Ma J, Yang H, Hu XH, Chen G: Prognostic Significance of LncRNA PVT1 and Its Potential Target Gene Network in Human Cancers: a Comprehensive Inquiry Based Upon 21 Cancer Types and 9972 Cases. Cell Physiol Biochem 2018, 46(2):591.

19. Huppi K, Volfovsky N, Runfola T, Jones TL, Mackiewicz M, Martin SE, Mushinski JF, Stephens R, Caplen NJ: The Identification of MicroRNAs in a Genomically Unstable Region of Human 
Chromosome 8q24. Molecular Cancer Research Mcr, 6(2):212-221.

20. Alvarez ML, Khosroheidari M, Eddy E, Kiefer J: Role of microRNA 1207-5P and its host gene, the long non-coding RNA Pvt1, as mediators of extracellular matrix accumulation in the kidney: implications for diabetic nephropathy. Plos One 2013, 8(10):e77468.

21. Li C, Lyu J, Meng QH: MiR-93 Promotes Tumorigenesis and Metastasis of Non-Small Cell Lung Cancer Cells by Activating the PI3K/Akt Pathway via Inhibition of LKB1 / PTEN / CDKN1A. Journal of Cancer 2017, 8(5):870-879.

22. Alvarez ML, DiStefano JK: Towards microRNA-based therapeutics for diabetic nephropathy. Diabetologia, 56(3):444-456.

23. Yang $Y$, Zhang $Y$, Xie A: Expression and significance of serum miRNA-93 in diabetic nephropathy patients. Experimental \& Laboratory Medicine 2012.

24. Long J, Wang Y, Wang W, Chang BHJ, Danesh FR: Identification of MicroRNA-93 as a Novel Regulator of Vascular Endothelial Growth Factor in Hyperglycemic Conditions. Journal of Biological Chemistry, 285(30):23457-23465.

25. Ma J, Zhang L, Hao J, Li N, Tang J, Hao L: Up-regulation of microRNA-93 inhibits TGF- $\beta 1$-induced EMT and renal fibrogenesis by down-regulation of Orai1. Journal of Pharmacological Sciences:S1347861318300033.

26. Livak KJ ST: Analysis of Relative Gene Expression Data Using Real-Time Quantitative PCR and the 2(-Delta Delta C(T))Method. METHODS 2001, Dec;25(4):402-408.

27. Li W, Ding Y, Smedley C, Wang Y, Chaudhari S, Birnbaumer L, Ma R: Increased glomerular filtration rate and impaired contractile function of mesangial cells in TRPC6 knockout mice. Sci Rep 2017, 7(1):4145.

28. Ding T, Chen W, Li J, Ding J, Mei X, Hu H: High Glucose Induces Mouse Mesangial Cell Overproliferation via Inhibition of Hydrogen Sulfide Synthesis in a TLR-4-Dependent Manner. Cell Physiol Biochem 2017, 41(3):1035.

29. Yan G, Chen ZY, Yan W, Yan L, Ma JX, Li YK: Long non-coding RNA ASncmtRNA-2 is upregulated in diabetic kidneys and high glucose-treated mesangial cells. Experimental \& Therapeutic Medicine 2017, 13(2):581-587.

30. Ai-Ling LI, Peng R, Sun Y, Peng HM, Hong Yl, Zhang Z: Effect of IncRNA-1700020114Rik on the fibrosis in mouse mesangial cells in high glucose concentration. Basic \& Clinical Medicine 2017.

31. Wang M, Yao D, Wang S, Yan Q, Lu W: Long non-coding RNA ENSMUST00000147869 protects mesangial cells from proliferation and fibrosis induced by diabetic nephropathy. Endocrine 2016, 54(1):1-12.

32. Wang M, Wang S, Yao D, Yan Q, Lu W: A novel long non-coding RNA CYP4B1-PS1-001 regulates proliferation and fibrosis in diabetic nephropathy. Molecular \& Cellular Endocrinology 2016, 426(C):136-145.

33. Yang YR, Zang SZ, Zhong CL, Li YX, Zhao SS, Feng XJ: Increased expression of the IncRNA PVT1 promotes tumorigenesis in non-small cell lung cancer. Int J Clin Exp Pathol 2014, 7(10):6929. 
34. Zhao L, Kong H, Sun H, Chen Z, Chen B, Zhou M: LncRNA-PVT1 promotes pancreatic cancer cells proliferation and migration through acting as a molecular sponge to regulate miR-448. $J$ Cell Physiol 2018, 233(5).

35. Huang C, Liu S, Wang H, Zhang Z, Yang Q, Gao F: LncRNA PVT1 overexpression is a poor prognostic biomarker and regulates migration and invasion in small cell lung cancer. Am J Trans/ Res 2016, 8(11):5025.

36. Zhao L, Kong H, Sun H, Chen Z, Chen B, Zhou M: LncRNA-PVT1 promotes pancreatic cancer cells proliferation and migration through acting as a molecular sponge to regulate miR-448. J Cell Physiol 2018, 233(5):4044-4055.

37. Alvarez ML, Distefano JK: Functional Characterization of the Plasmacytoma Variant Translocation 1 Gene (PVT1) in Diabetic Nephropathy. PLOS ONE 2011, 6(4).

38. Huppi K, Volfovsky N, Runfola T, Jones TL, Mackiewicz M, Martin SE, Mushinski JF, Stephens R, Caplen NJ: The identification of microRNAs in a genomically unstable region of human chromosome 8q24. Mol Cancer Res 2008, 6(2):212.

39. Zhang L, Liang X, Li Y: Long non-coding RNA MEG3 inhibits cell growth of gliomas by targeting miR93 and inactivating PI3K/AKT pathway. Oncol Rep 2017, 38(4):2408.

40. Badal SS, Yin W, Long J, Corcoran DL, Chang BH, Luan DT, Kanwar YS, Overbeek PA, Danesh FR: miR-93 regulates Msk2-mediated chromatin remodelling in diabetic nephropathy. Nat Commun 2016, 7:12076.

41. Zou HL, Wang Y, Gang Q, Zhang Y, Sun Y: Plasma level of miR-93 is associated with higher risk to develop type 2 diabetic retinopathy. Graefes Arch Clin Exp Ophthalmol 2017, 255(6):1-8.

42. Huang C, Lin MZ, Cheng D, Filip B, Pollock CA, Chen XM: KCa3.1 mediates dysfunction of tubular autophagy in diabetic kidneys via PI3k/Akt/mTOR signaling pathways. Sci Rep 2016, 6:23884.

43. Mavroeidi V, Petrakis I, Stylianou K, Katsarou T, Giannakakis K, Perakis K, Vardaki E, Stratigis S, Ganotakis E, Papavasiliou S: Losartan affects glomerular AKT and mTOR phosphorylation in an experimental model of type 1 diabetic nephropathy. J Histochem Cytochem 2013, 61(6):433-443.

44. Lu Q, Zuo WZ, Ji XJ, Zhou YX, Liu YQ, Yao XQ, Zhou XY, Liu YW, Yin XX: Ethanolic Ginkgo biloba leaf extract prevents renal fibrosis through Akt/mTOR signaling in diabetic nephropathy. Phytomedicine 2015, 22(12):1071-1078.

45. Du J, Liu S, He J, Liu X, Qu Y, Yan W, Fan J, Li R, Xi H, Fu W: MicroRNA-451 regulates stemness of side population cells via PI3K/Akt/mTOR signaling pathway in multiple myeloma. Oncotarget 2015, 6(17):14993-15007.

46. Li NA, Wang W, Xu B, Gong H: miR-196b regulates gastric cancer cell proliferation and invasion via PI3K/AKT/mTOR signaling pathway. Oncol Lett 2016, 11(3):1745-1749.

\section{Tables}

Table 1 The sequences of siRNAs and primers 


\begin{tabular}{cl}
\hline Primer & \multicolumn{1}{c}{ Sequence } \\
\hline siPVT1 & Forwrad:5'-GCUUGGAGGCUGAGGAGUUTT-3' \\
siNC & Reverse:5'-AACUCCUCAGCCUCCAAGCTT-3' \\
& Forwrad:5'-UUCUCCGAACGUGUCACGUTT-3' \\
& Reverse:5'-ACGUGACACGUUCGGAGAATT-3' \\
\hline LncRNA-PVT1 & Forward: 5'-ATTGAGATGTGAAGCGTTGA-3' \\
& Reverse: 5'-AGGCACCTTTCCCAGTT-3' \\
\hline CyclinD1 & Forward: 5'-AGCTCCTGTGCTGCGAAGTGGA-3' \\
& Reverse: 5'-AGTGTTCAATGAAATCGTGCGGG-3' \\
\hline CDK4 & Forward: 5'-AGTAATGGGACCGTCAAGC-3' \\
& Reverse: 5'-CACCAAGACTGGGAAAGG-3' \\
\hline miR-93-5P & Forward: 5'-AGGCCCAAAGTGCTGTTCGT-3' \\
& Reverse: 5'- GTGCAGGGTCCGAGG-3' \\
\hline U6 & Forward:5'-CTCGCTTCGGCAGCACA-3' \\
& Reverse: 5'-AACGCTTCACGAATTTGCGT-3' \\
\hline & Forward: 5'-TGACGTGCCGCCTGGAGAAAC-3' \\
\hline & \\
\hline &
\end{tabular}

Figures 
A

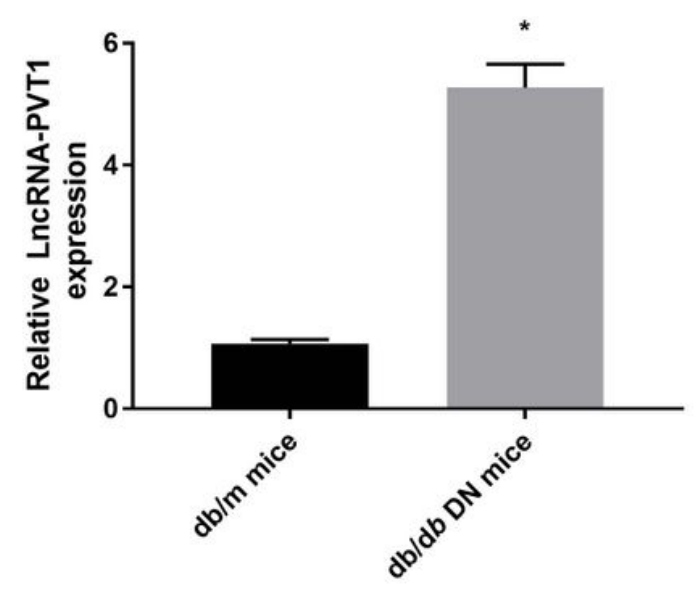

B

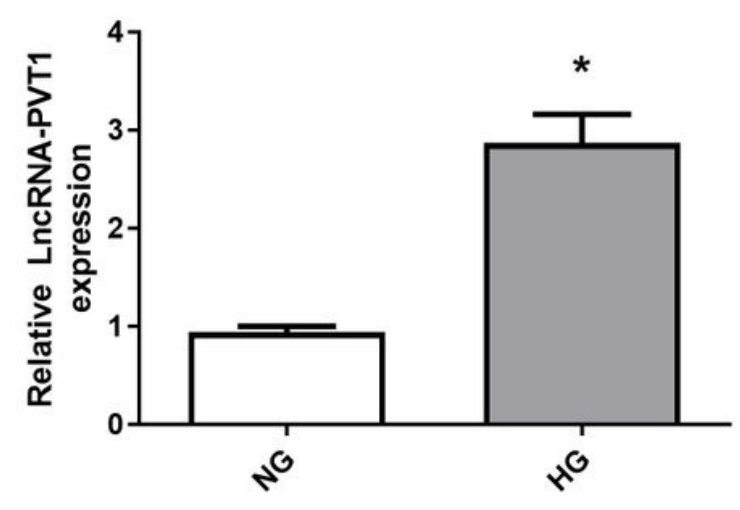

\section{Figure 1}

Expression of LncRNA-PVT1 in kidney tissues and mouse mesangial cells (MMCs). A, qRT-PCR was used to detect the expression of LncRNA-PVT1 in kidney tissues of $\mathrm{db} / \mathrm{m}$ (Normal) and db/db (DN) mice; *, P< 0.05 compared with the $\mathrm{db} / \mathrm{m}$ mice. $\mathrm{B}$, qRT-PCR was used to detect the expression of LncRNA-PVT1 in MMCs under normal glucose (NG) and high glucose $(\mathrm{HG})$ condition; *, $\mathrm{P}<0.05$ compared to the NG group. 
A

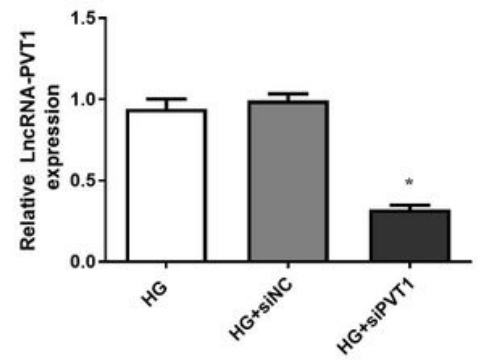

C
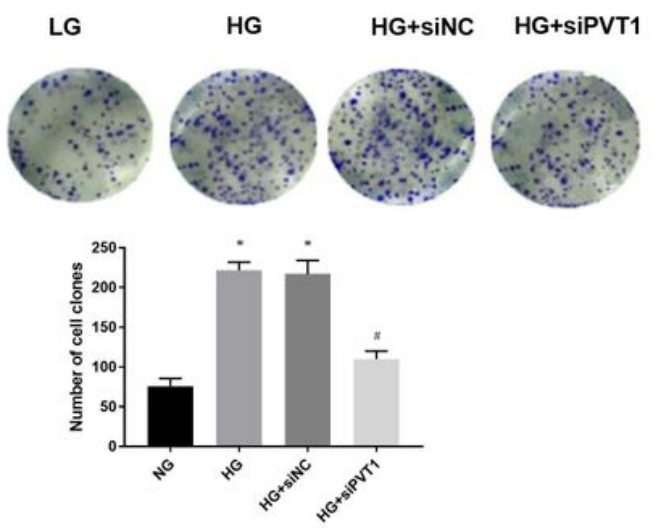

E

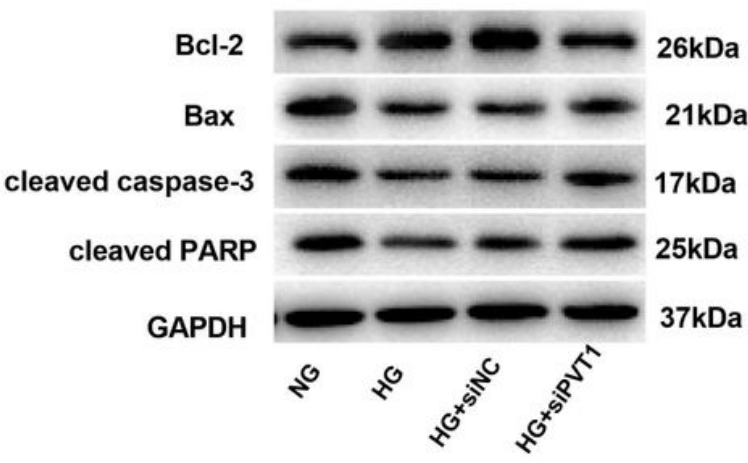

B
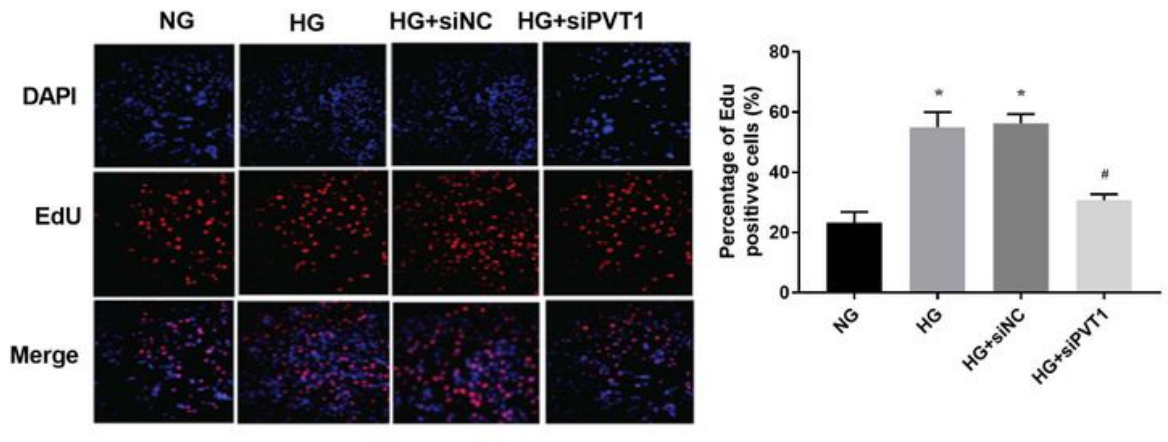

D

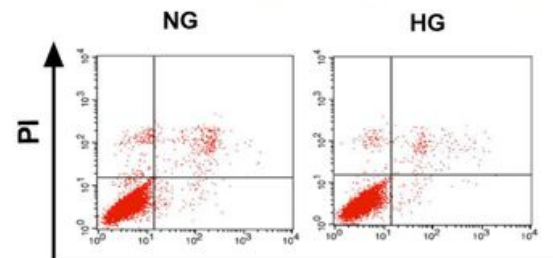

HG+siNC
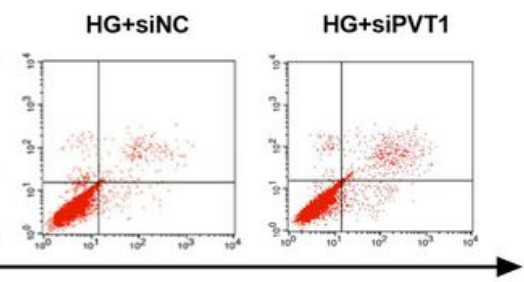

Annexin V

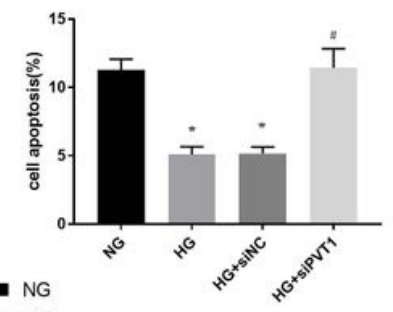

- NG

II HG

HG+siPVT1

\section{Figure 2}

Effects of IncRNA-PVT1 on the proliferation and apoptosis of mouse mesangial cells (MMCs) under high glucose (HG) condition. A, RNA interference efficiency; * P < 0.05 compared with the HG and HG + siPVT1 group. B, EdU assay was used to detect the proliferation ability $(\times 200)$. C, Colony formation assay was used to detect the colony forming ability. D, Flow cytometry was used to detect the apoptosis rate. $\mathrm{E}$, Western blot was used to detect the expression of apoptotic proteins $(\mathrm{Bcl}-2$, Bax, cleaved caspase-3, cleaved PARP). * $\mathrm{P}<0.05$ compared with NG group; \#, $\mathrm{P}<0.05$ compared with the HG and HG + siPVT1 group. The data among multi-groups were analyzed by One-Way ANOVA, followed by Tukey's test (between two groups). 
A
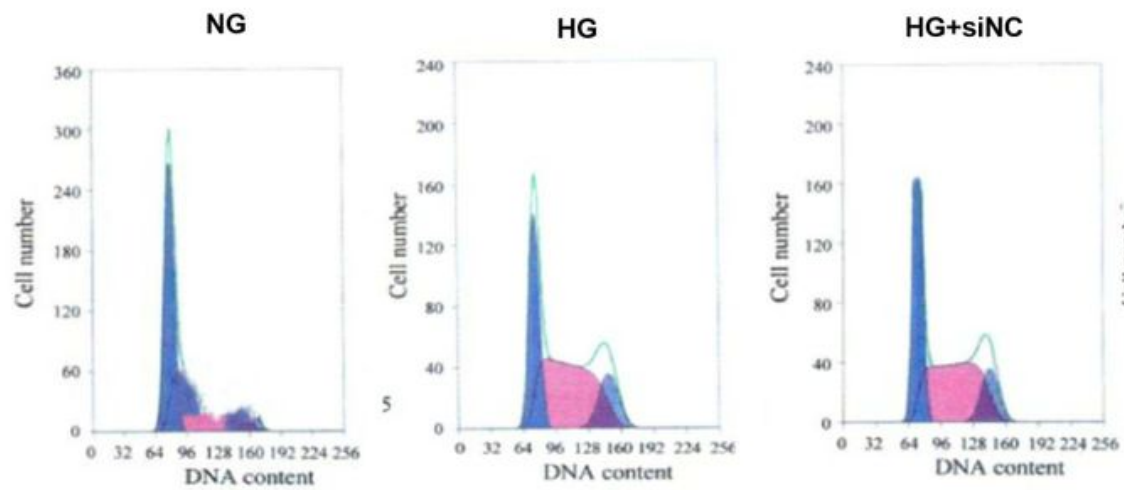

HG+siPVT1

B

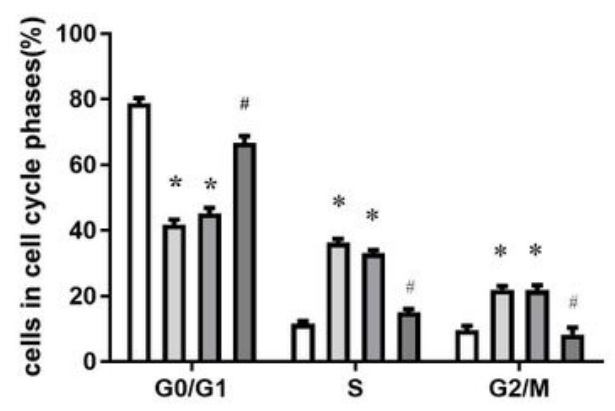

C

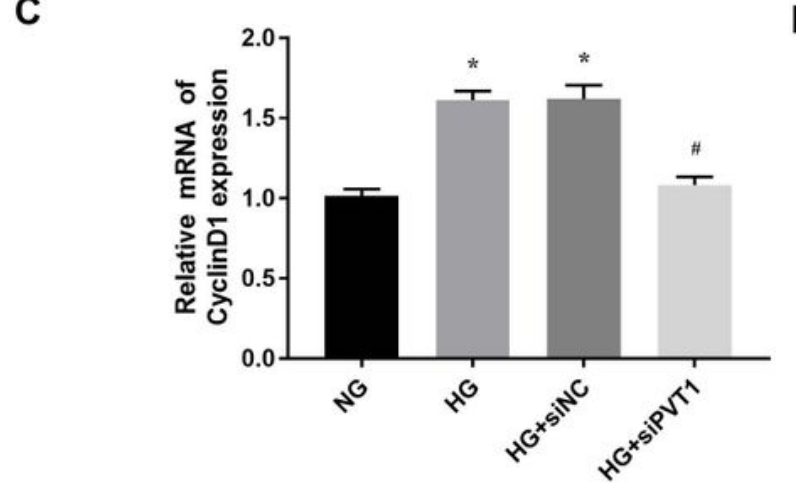

E

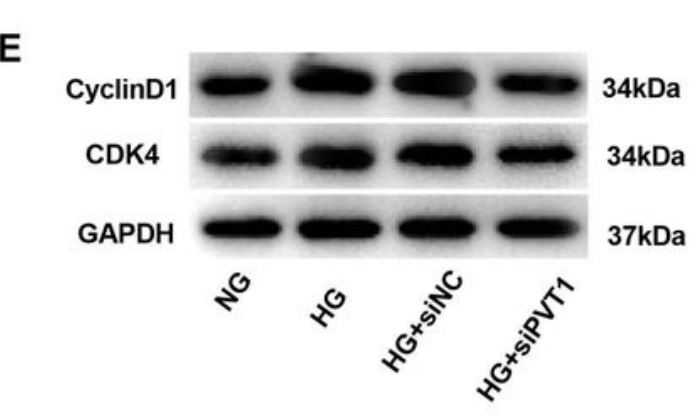

D

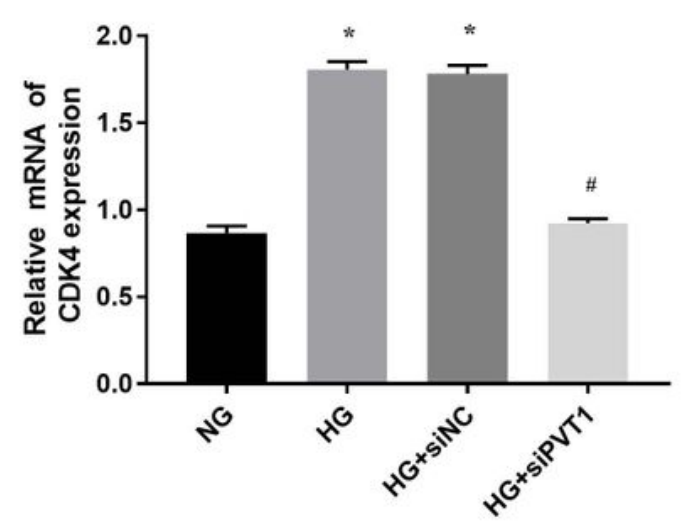

口 NG

口 HG

口 $\mathrm{HG}+\operatorname{sinC}$

口 HG+siPVT1

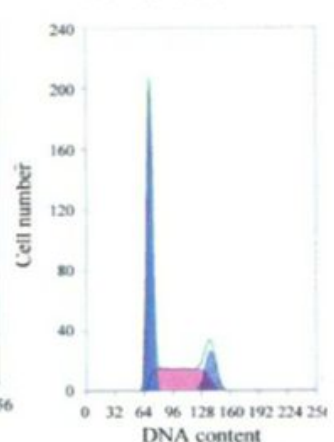

DNA content

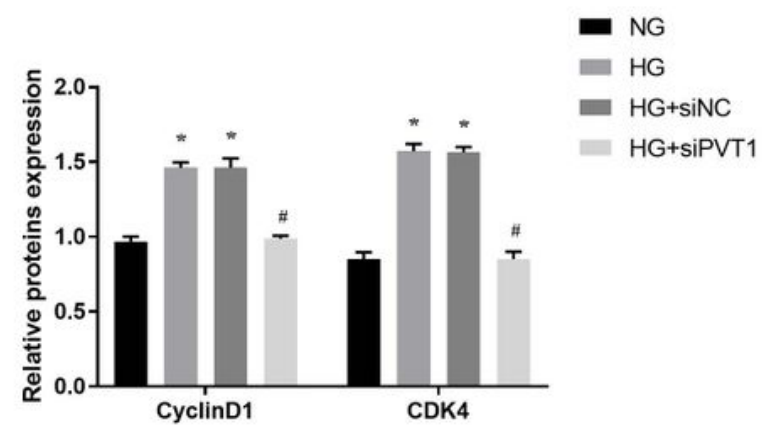

\section{Figure 3}

Effect of IncRNA-PVT1 on the cell cycle of mouse mesangial cells (MMCs) under high glucose (HG) condition. A, Flow cytometry was used to detect the cell cycle. B, the percentages of cells in different cell cycle phases. C, qRT-PCR was used to detect the expression of CyclinD1. D, qRT-PCR was used to detect the expression of CDK4. E, Western blot was used to detect the expression of CyclinD1 and CDK4. *, P< 
0.05 compared with the NG group; \#, P < 0.05 compared with the HG and HG + siPVT1 group. The data among multi-groups were analyzed by One-Way ANOVA, followed by Tukey's test (between two groups).

A

NG

HG

HG+siNC HG+siPVT1

oh
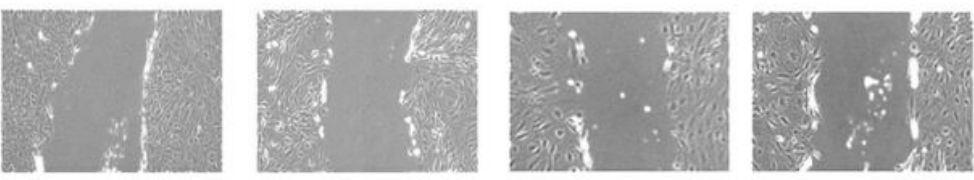

48h
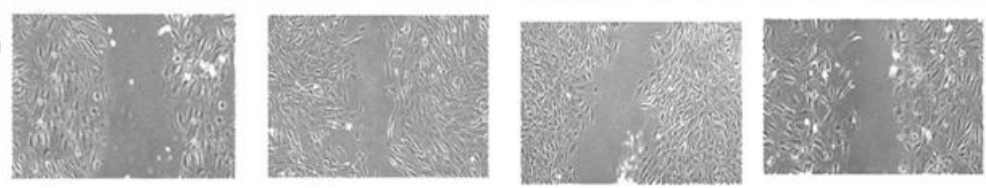

B
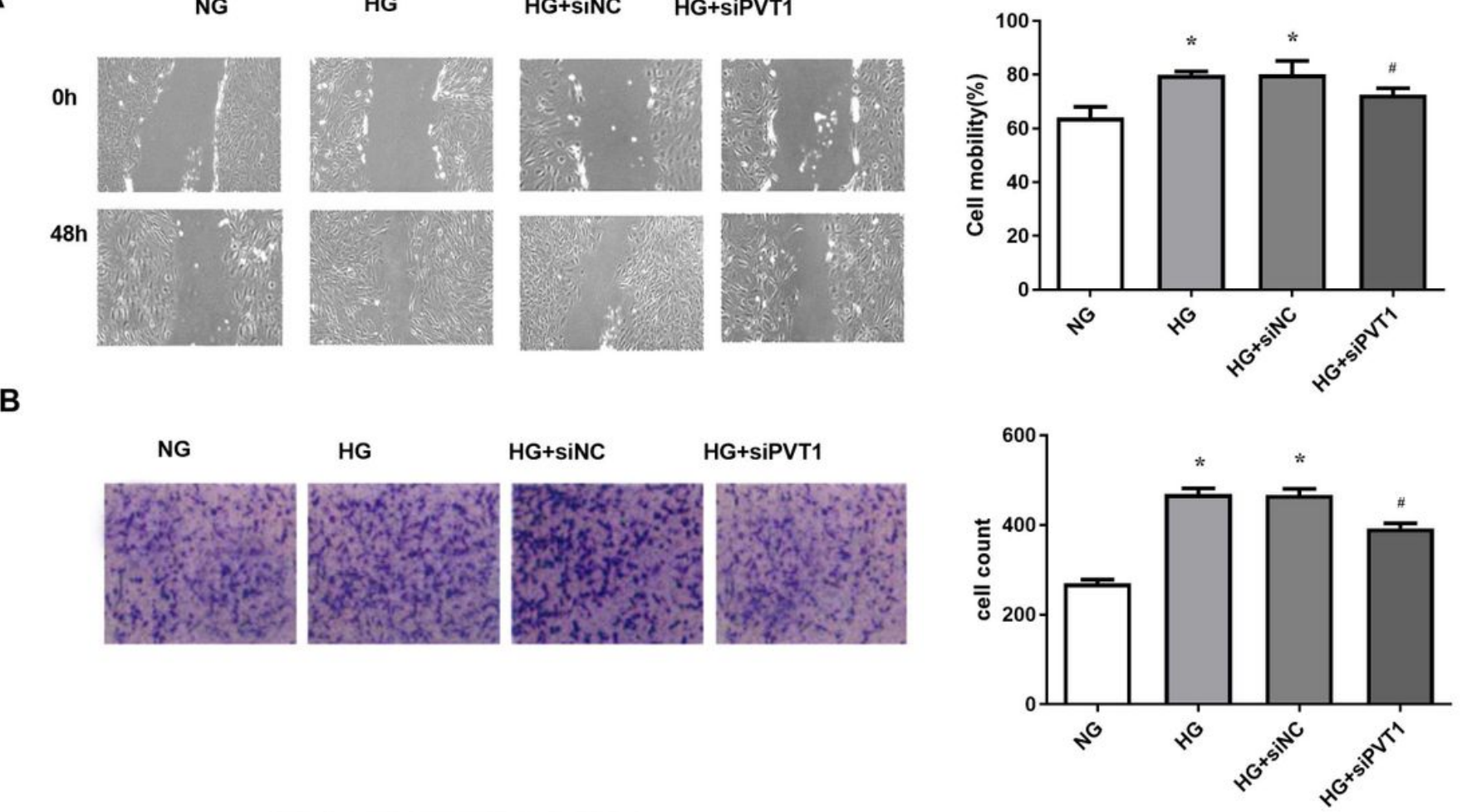

C
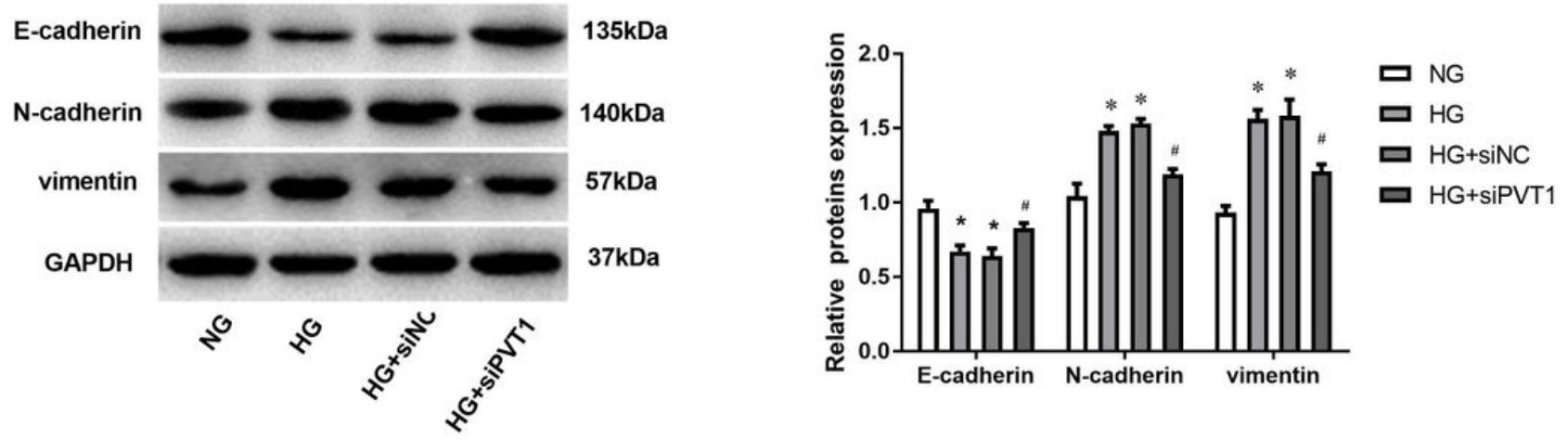

Figure 4

Effects of IncRNA-PVT1 on the migration and invasion of mouse mesangial cells (MMCs) under high glucose $(\mathrm{HG})$ condition. A, Scratch assay was used to detect the migration ability $(\times 200)$. B, Transwell assay was used to detect the invasion ability ( $\times 200)$. C, Western blot was used to detect the expression of EMT proteins ( $\mathrm{N}$-cadherin, vimentin, and E-cadherin). ${ }^{*}, \mathrm{P}<0.05$ compared with the NG group; \#, $\mathrm{P}<$ 0.05 compared with the HG and HG + siPVT1 group. The data among multi-groups were analyzed by OneWay ANOVA, followed by Tukey's test (between two groups). 
A

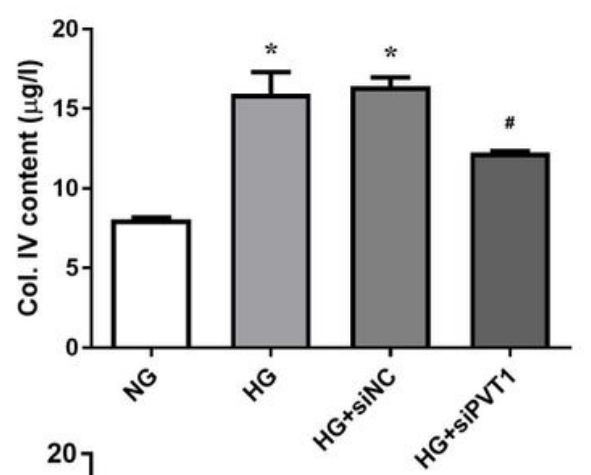

C

E
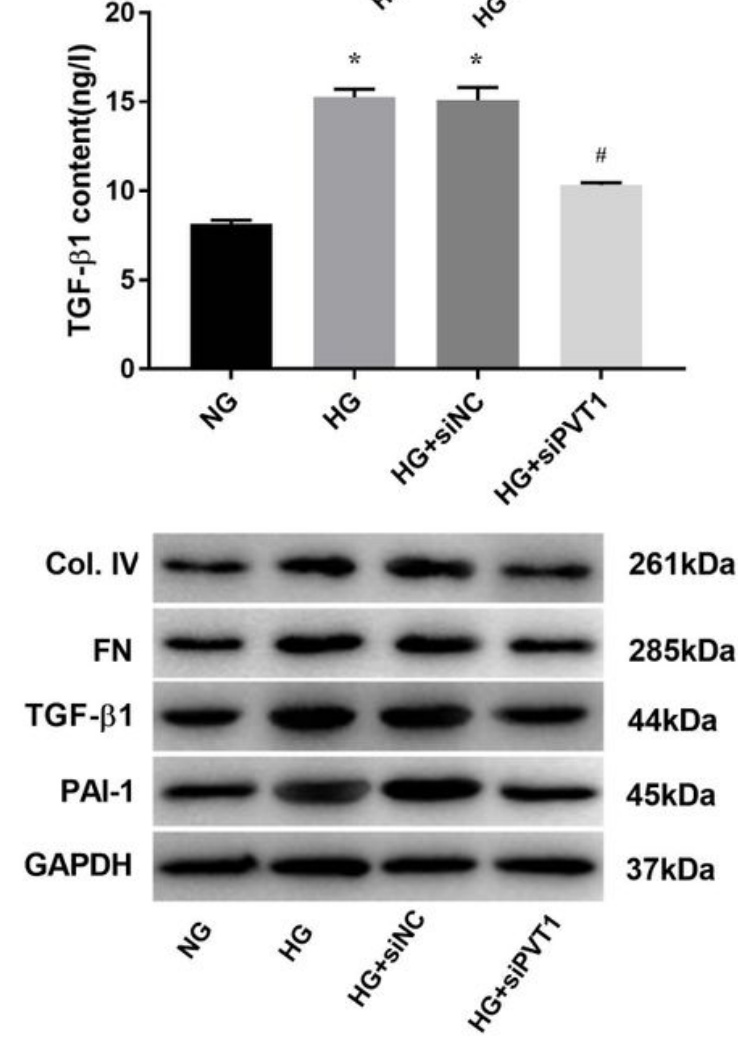

B

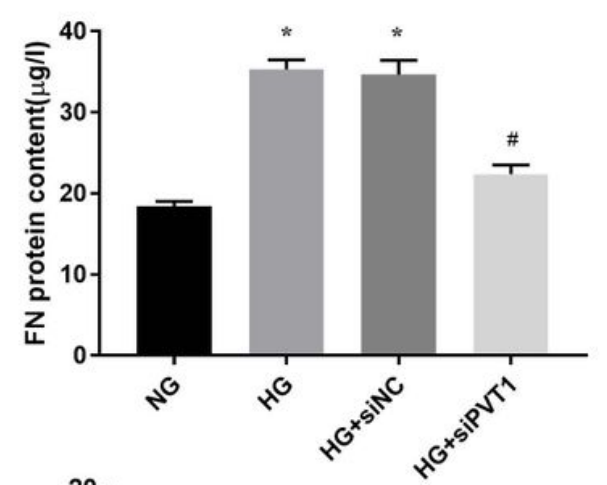

D
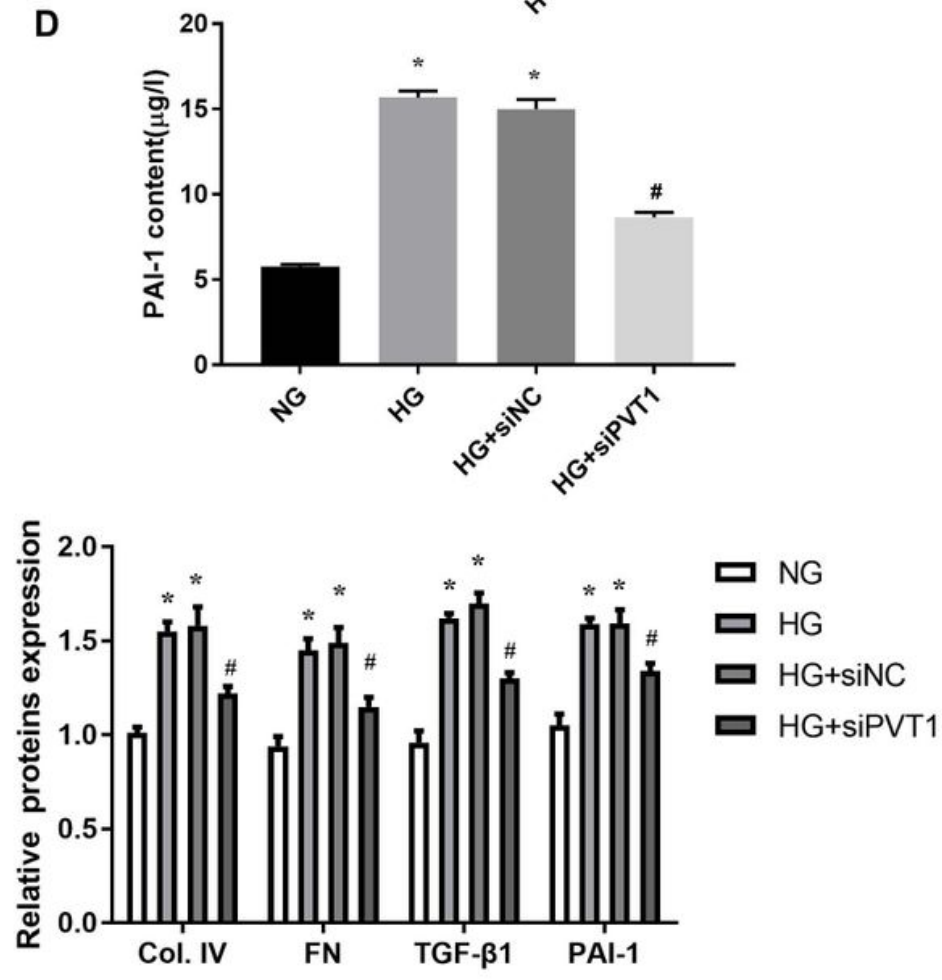

Figure 5

Effect of IncRNA-PVT1 on the fibrosis of mouse mesangial cells (MMCs) under high glucose (HG) condition. A-D, ELISA was used to detect the contents of fibrosis proteins (Col. IV, FN, TGF- $\beta 1$, and PAl-1). $E$, Western blot was used to detect the expression of fibrosis proteins. ${ }^{*}, \mathrm{P}<0.05$ compared with the NG group; \#, P $<0.05$ compared with the HG and HG + siPVT1 group. The data among multi-groups were analyzed by One-Way ANOVA, followed by Tukey's test (between two groups). 
A

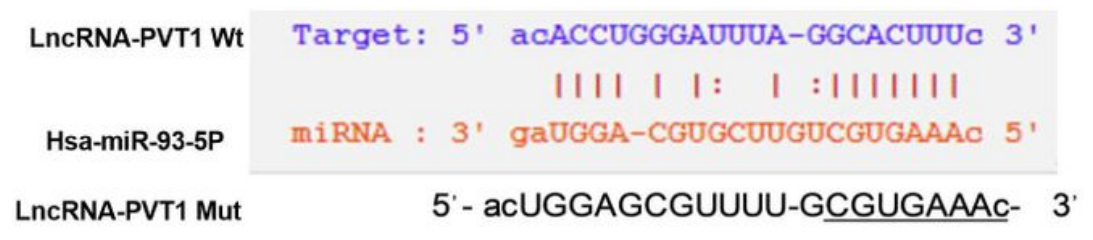

C

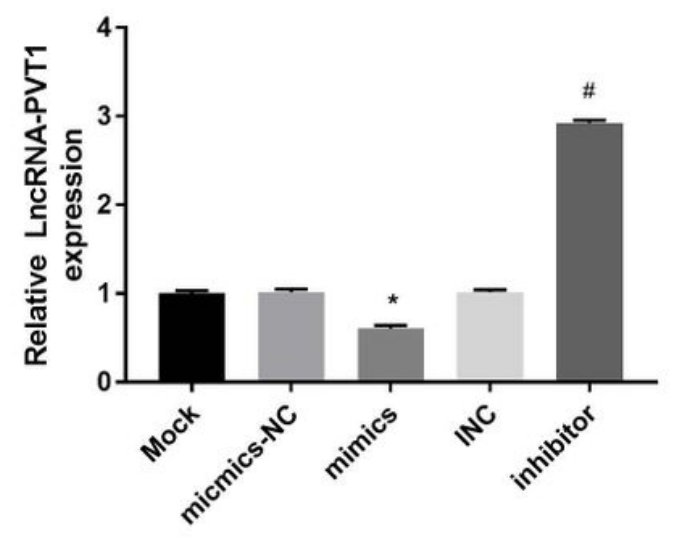

B

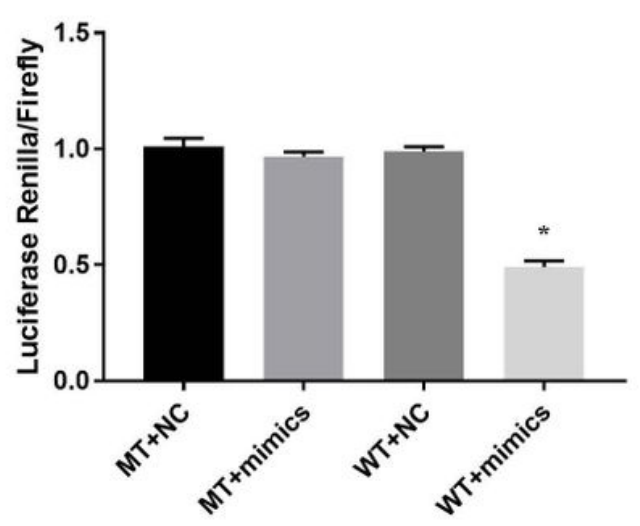

D

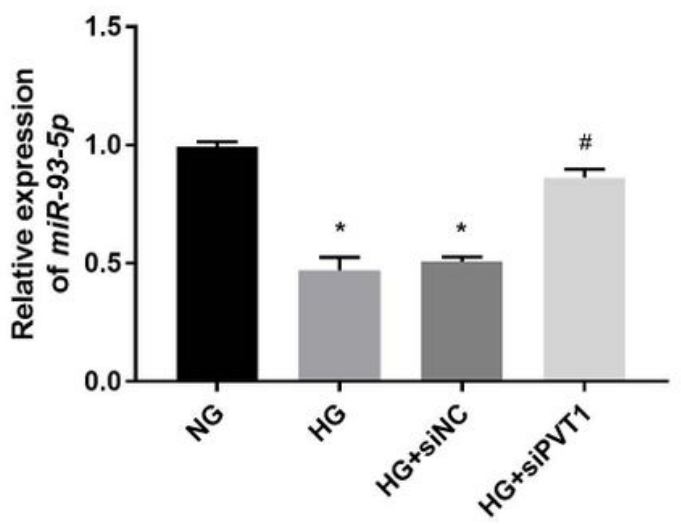

Figure 6

MiR-93-5p was the target gene of LncRNA-PVT1. A, StarBase3.0 software was used to predict the binding site of miR-93-5p to PVT1. B, Dual luciferase reporter gene assay was used to identify the relationship between miR-93-5p and PVT1; *, P $<0.05$ compared with the WT + NC group. C, qRT-PCR was used to detect the expression of PVT1 in cells transfected with miR-93-5p mimics or inhibitor; *, $\mathrm{P}<0.05$ compared with the Mock and mimics-NC group; \#, $\mathrm{P}<0.05$ compared with the Mock and INC group. D, qRT-PCR was used to detect the expression of miR-93-5p in cells transfected with siPVT1; ${ }^{*}, \mathrm{P}<0.05$ compared with the NG group; \#, P $<0.05$ compared with the HG and HG + siPVT1 group. The data among multi-groups were analyzed by One-Way ANOVA, followed by Tukey's test (between two groups). 
A

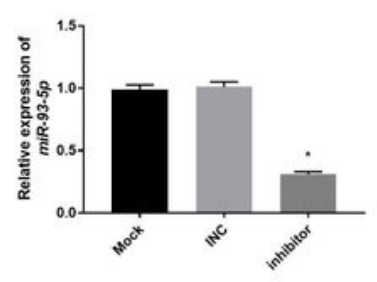

C

Oh

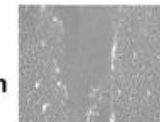

$48 \mathrm{~h}$
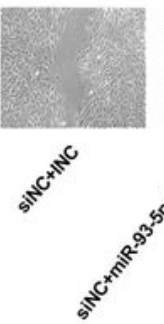

D
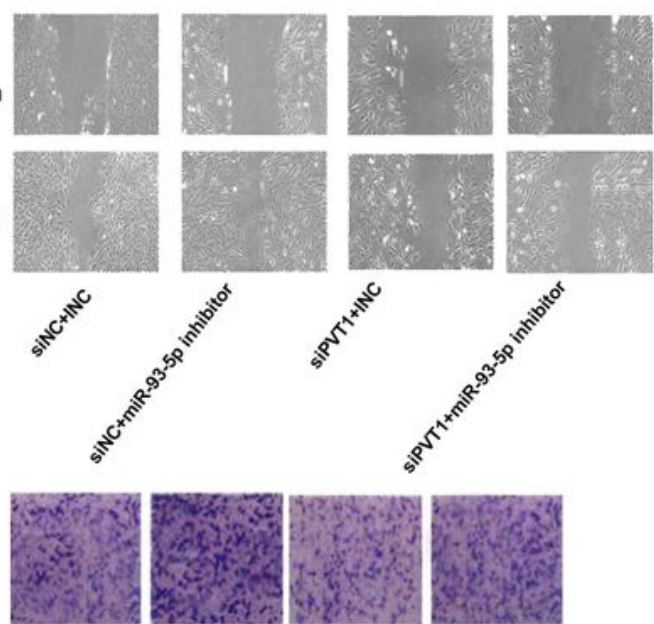

B
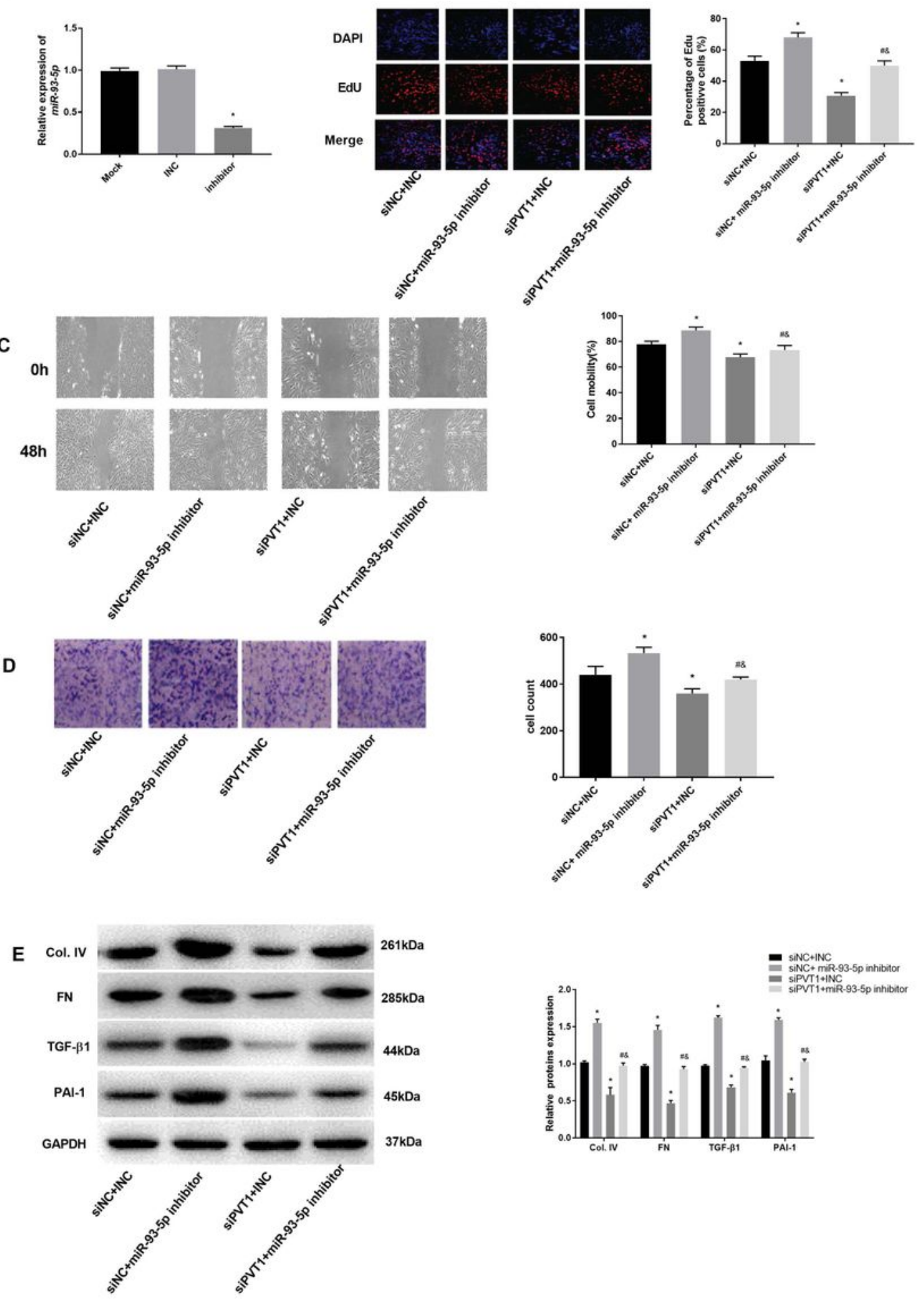

\section{Figure 7}

MiR-93-5p eliminated the effect of LncRNA-PVT1 in mouse mesangial cells (MMCs) under high glucose (HG) condition. A, the transfection efficiency of miR-93-5p inhibitor; *, $\mathrm{P}<0.05$ compared with the Mock and INC group. B, EdU assay was used to detect the proliferation ability (× 200). C, Scratch assay was used to detect the migration ability. D, Transwell assay was used to detect the invasion ability $(\times 200)$. E, Western blot was used to detect the expression of fibrosis proteins. *, $\mathrm{P}<0.05$ compared with the siNC + 
INC group; \#, P $<0.05$ compared with the siNC + miR-93-5p inhibitor group; \&, P $<0.05$ compared with the siPVT1 + INC group. The data among multi-groups were analyzed by One-Way ANOVA, followed by Tukey's test (between two groups).

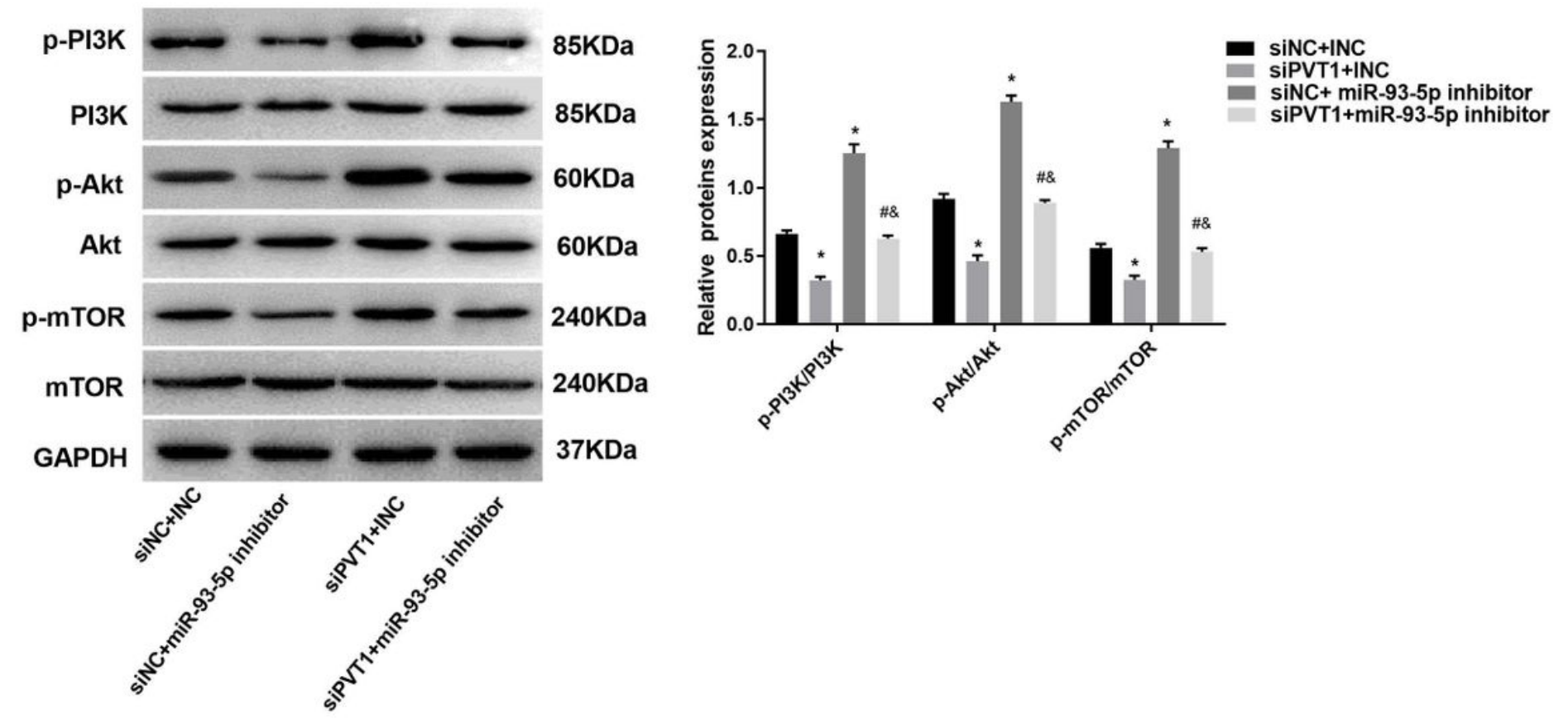

Figure 8

LncRNA-PVT1 and miR-93-5p mediated the PI3K/Akt/mTOR pathway in mouse mesangial cells (MMCs) under high glucose $(\mathrm{HG})$ condition. Western blot was used to detect the expression of PI3K/Akt/mTOR proteins (p-PI3K/PI3K, p-Akt/Akt, and p-mTOR/mTOR). * $\mathrm{P}<0.05$ compared with the siNC + INC group; \#, $P<0.05$ compared with the siNC + miR-93-5p inhibitor group; \&, P $<0.05$ compared with the siPVT1 + INC group. The data among multi-groups were analyzed by One-Way ANOVA, followed by Tukey's test (between two groups).

\section{Supplementary Files}

This is a list of supplementary files associated with this preprint. Click to download.

- NC3RsARRIVEGuidelinesChecklistfillable.pdf 